\title{
Modelling dielectric and magnetic properties of ferroconcrete
}

\author{
T. Frenzel and M. Koch
}

Leibniz University Hannover, Institute for Electrical Engineering and Measurement Science, Appelstraße 9A, 30167

Hannover, Germany

\begin{abstract}
This contribution discusses the modelling and parameterization of dielectric and magnetic properties of ferroconcrete by using numerical electromagnetic field analysis software. The software is based on the Method of Moments (MoM). The shielding effectiveness (SE) of the ferroconcrete DUT was already measured in a study by order of the government. According to these results, the ferroconcrete DUT is modelled and calculated. Therefore the DUT is subdivided into two parts. The first part represents the reinforcement mesh; the second part represents the lossy concrete with complex permittivity. Afterwards, the reflection and transmission properties of numerical analysed building materials are validated and compared with the measurement results in a frequency range of $30-1000 \mathrm{MHz}$.
\end{abstract}

\section{Introduction}

In a study by order of the German federal office for the security of the information technology (BSI) the shielding effectiveness (SE) of several building materials have been measured. The measurements are carried out in a fully anechoic room in accordance to the IEEE 299, 1997,(IEEE, 1998). In Frenzel et al. (2007) results of the measurement of four DUTs (DUT) are already presented and validated. The DUTs are constructed like walls with dimensions of $2 \mathrm{~m} \times 2 \mathrm{~m}$ and are applied at a specially designed anechoic chamber. The anechoic chamber in the real measurement setup is necessary to ensure field incidence only through the shield. Reflected and refracted parts of the field are shielded by the anechoic chamber. Furthermore, the anechoic chamber is fitted with absorbers to minimize resonances.

In the following, it is investigated, if the SE of the measured DUTs and of the anechoic chamber can be analytical or numerical simulated. Therefore, the ferroconcrete DUT is subdivided into two parts. The first part represents the reinforcement mesh; the second part represents the lossy con-

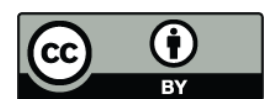

Correspondence to: T. Frenzel

(frenzel@ieee.org)

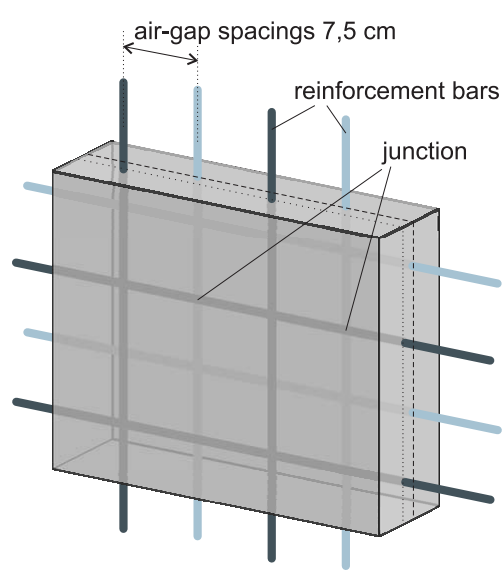

Fig. 1. Schematically representation of ferroconcrete.

crete with complex permittivity. The reinforcement mesh of the ferroconcrete DUT will be analytically modelled by Shelkunoff estimation. The concrete is modelled in an electromagnetic field analysis tool based on the MoM. For the numerical analysis, a models of the anechoic chamber is considered, on which the DUT is applied. In this model the chamber is simulated by a plane with dimensions that are large relatively to the wavelength.

The electromagnetic field and the resulting SE will be calculated according to (IEEE, 1998). As shown in Frenzel et al. (2007), the reinforcement mesh of ferroconcrete has a high SE in a frequency range below his cutoff frequency. However, the SE is rising for higher frequencies due to the loss attenuation of concrete. Thus, the complex permittivity of the concrete part is estimated. Finally, the results of the real measurements, the analytical analysis and the numerical calculations are compared and validated.

\section{Description of ferroconcrete}

The ferroconcrete DUT is composed of standard concrete and steel reinforcement bars. The reinforcement is double arranged in a manner to minimize the resulting air-gap spacing.

Published by Copernicus Publications on behalf of the URSI Landesausschuss in der Bundesrepublik Deutschland e.V. 


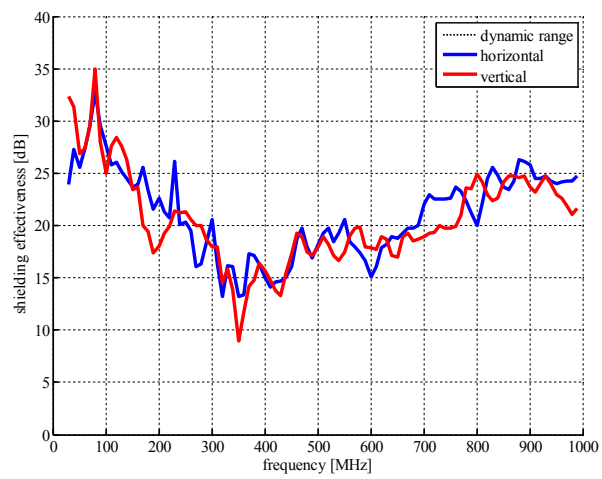

Fig. 2. Shielding effectiveness of ferroconcrete.

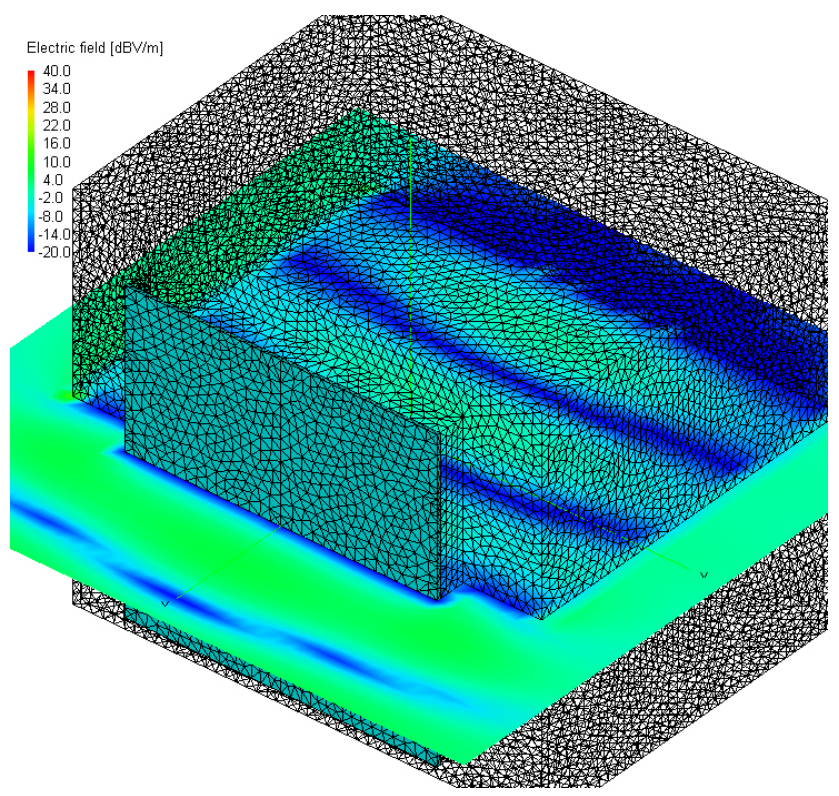

Fig. 3. Model of the anechoic chamber.

The distance between two bars is $7.5 \mathrm{~cm}$. This concrete's type is the common $\mathrm{C} 25 / 30 \mathrm{XC} 3$ with defined composition of cement, sand, gravel and water. In this case, the moisture content of dry concrete after the complete hydration process shall be about 0.14 . Due to a short-term storage of six months for the ferroconcrete the moisture content is expected much higher. The Fig. 1 shows a schematic representation of the ferroconcrete and its measured SE.

\section{Modelling Measurement Setup}

The real measurements are carried out in accordance to IEEE 299-1997 in a fully anechoic room (FAR). A specially designed anechoic chamber is used to ensure field incidence only through the DUT. Furthermore, the anechoic chamber is fitted with absorbers to minimize resonances. For the measurement setup, the emitting antenna is located outside the

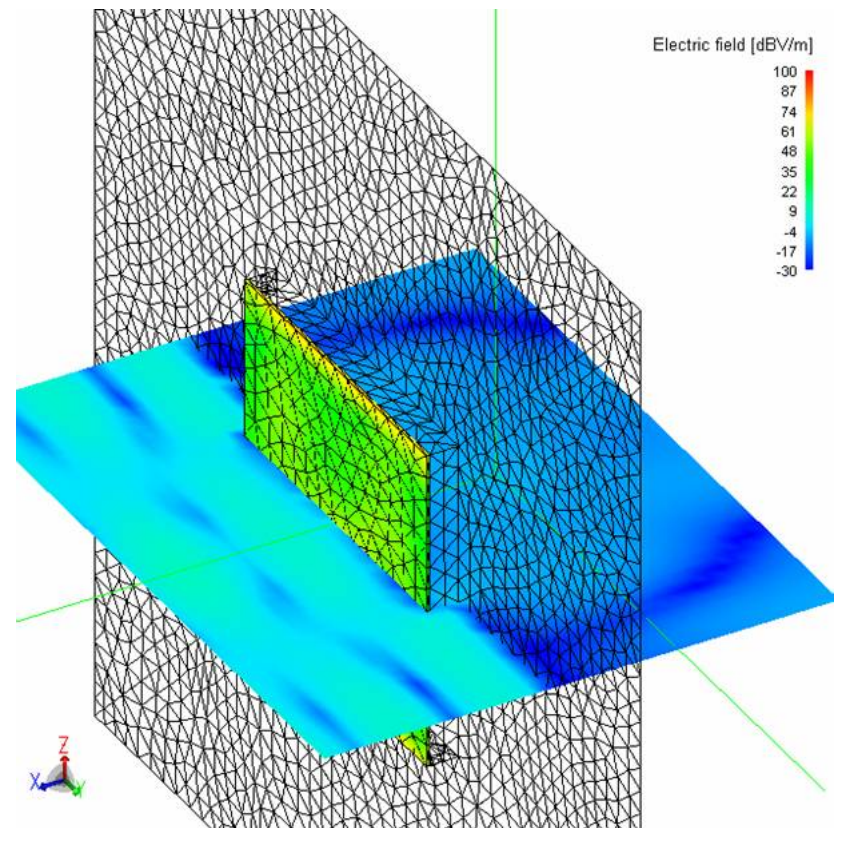

Fig. 4. Model of the infinite plane.

anechoic chamber in a distance to ensure far field conditions. The receiving antenna is located inside the chamber. At a window in front of the chamber the ferroconcrete DUT is applied. The complete measurement setup has been already presented in Frenzel et al. (2007).

Concerning the maximum frequency of $1000 \mathrm{MHz}$, the measurement setup is very large. Due to that fact field calculations are made with the Method of Moments (MoM). For modelling the anechoic chamber two alternatives are considered. Firstly, an ideal replication of the anechoic chamber without absorbers is modelled (cp. Fig. 2). The walls are perfectly electric conducting (PEC). Thus, resonances occur at frequencies in agreement with the measurements. However, it is modelled without absorbing material inside. Advantages of this model are high shielding against deflected and diffracted waves. Disadvantages of this model are high resonances in the considered frequency range.

The second model of the anechoic chamber (cp. Fig. 3) is a perfect electric conducting (PEC) plane with dimensions that a large relatively to the wavelength. In particular, the plane is four or more times the wavelength. Advantage of this model is the prevention of cavity resonances without the modelling of absorbing material. However, the plane must be very large to avoid edge diffraction. First results show, that the plane model ensures a dynamic range of the SE up to $40 \mathrm{~dB}$.

The frequency range for the calculation is between $30 \mathrm{MHz}$ and $1000 \mathrm{MHz}$ and therefore the imaginary permittivity is calculated to $\varepsilon_{r, 30 \mathrm{MHz}}^{\prime \prime}=0.709$ for a frequency of $f=30 \mathrm{MHz}$ and to $\varepsilon_{r, 1000 \mathrm{MHz}}^{\prime \prime}=21.69$ for a frequency of $f=1000 \mathrm{MHz}$. The real part of the permittivity is between 


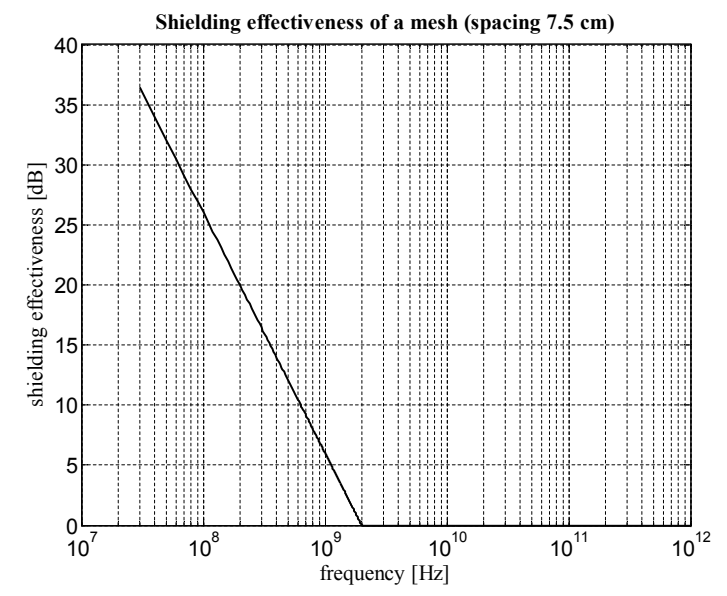

Fig. 5. Shielding effectiveness of a mesh; $30 \mathrm{MHz}-1 \mathrm{THz}$ [3].

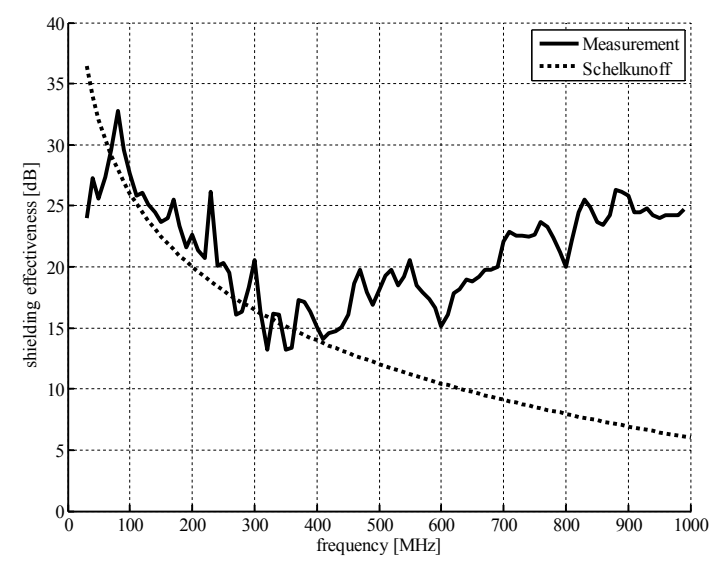

Fig. 6. Shielding effectiveness of the mesh in comparison with measurement results.

$\varepsilon_{r, 30 \mathrm{MHz}}^{\prime}=80.99$ and $\varepsilon_{r, 30 \mathrm{MHz}}^{\prime}=74.51$. In the numerical model these permittivity is implemented and results in a calculated field strength and SE respectively (cp. Fig. 6).

According to the measurement setup, both models allow plane wave (TEM) excitation. In the far field, the interdependence of the electric and magnetic field strength vector is based on the characteristic impedance of a plane wave in vacuum $Z_{0}=120 \pi \Omega$. Therefore, only the amplitude of the incident electric field strength is determined.

\section{Modelling ferroconcrete}

As shown at the beginning ferroconcrete consists of two basic components. Firstly, a mesh of reinforcement bars; secondly, a body of concrete. The modelling of a conducting mesh offers an analytically estimation of the SE in accordance to Gonschorek (2005). In consideration of the real bar

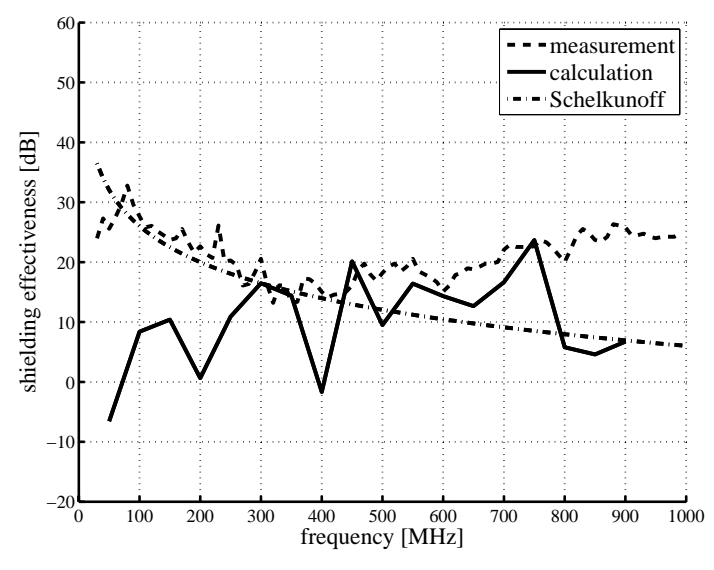

Fig. 7. Calculated shielding effectiveness of concrete without reinforcement.

dimensions Schelkunoff calculates the SE for meshes with maximum air-gap spacing $h$ to:

$a_{S} \approx 20_{10} \log \left(\frac{\lambda / 2}{h}\right) d B$; with $\lambda / 100<h<\lambda / 2$.

The SE of the presented reinforcement with air-gap spacing of $h=7.5 \mathrm{~cm}$ is estimated as presented in Fig. 4 and Fig. 5. The curve shows a distinct approximation of the measurements results and the estimation between $30 \mathrm{MHz}$ and $400 \mathrm{MHz}$. In the frequency range above $400 \mathrm{MHz}$ the measured curve rises up to $25 \mathrm{~dB}$, while the estimated curve according to Schelkunoff is decreasing. The decreasing is caused by the reinforcement's cutoff-frequency calculated to $f_{\text {cut }}(7.5 \mathrm{~cm})=2 \mathrm{GHz}$.

The rise of the measured curve must be caused by lossy parts of the concrete's permittivity. As shown in Kupfer (2000) rising moisture content of lime bricks rises the attenuation as well. In particular, the adsorbed water or free water layers of lime bricks lead to higher parts of the real and imaginary permittivity and higher conductivity. Following (Kraszewski, 1996) modelling the electromagnetic properties of concrete is considered as a mixture with one component being liquid water and being other components of sand, cement, gravel for example. The approach of this paper is to model the mixture of concrete dominating by the content of physically bounded and free water.

The frequency range for the calculation is between $30 \mathrm{MHz}$ and $1000 \mathrm{MHz}$ and therefore the imaginary permittivity is calculated to $\varepsilon_{r, 30 \mathrm{MHz}}^{\prime \prime}=0.709$ for a frequency of $f=30 \mathrm{MHz}$ and to $\varepsilon_{r, 1000 \mathrm{MHz}}^{\prime \prime}=21.69$ for a frequency of $f=1000 \mathrm{MHz}$. The real part of the permittivity is between $\varepsilon_{r, 30 \mathrm{MHz}}^{\prime}=80.99$ and $\varepsilon_{r, 30 \mathrm{MHz}}^{\prime}=74.51$. In the numerical model these permittivity is implemented and results in calculated field strength and SE respectively (cp. Fig. 6). 


\section{Results}

In this paper, a model of a measurement setup up is presented, consisting of a ferroconcrete DUT and an anechoic chamber. With this model the field strength and SE is calculated with a numerical field simulation tool using the Method of Moments (MoM). The numerical model must simulate the properties of the anechoic chamber and of the DUT as well.

The model of the ferroconcrete DUT is subdivided into two parts. The first part represents the reinforcement. The reinforcement is modelled by an analytical estimation of a mesh according to Schelkunoff (Frenzel et al., 2007). This estimation shows a significant agreement between the measurement and the calculation from $30 \mathrm{MHz}$ up to $400 \mathrm{MHz}$. The second part is represented by a thin layer of free water. The real and imaginary parts of permittivity are implemented in the model. The agreement of the measured and calculated SE is less satisfactory. However the result is a rising SE for higher frequencies. This trend agrees to the measurement results.

\section{References}

IEEE 299: Standard Method for Measuring the Effectiveness of Electromagnetic Shielding Enclosures, 50 pp., 1997.

IEEE: EMC Society, 39 pp., 1998.

Frenzel, T., Rohde, J., and Opfer, J.: Elektromagnetische Schirmung von Gebäuden, BSI, Bonn, 86 pp., 2007.

Gonschorek, K. H.: EMV für Geräteentwickler und Systemintegratoren, Berlin, Heidelberg, Springer-Verlag, 447 pp., 2005.

Kupfer, K.: Sensors Update: RF and Microwave Sensing of Moist Materials, 7, Weinheim, Wiley-VCH, 104 pp., 2000.

Kraszewski, A.: Microwave Aquametry: Electromagnetic Wave Interaction with Water-Containing Materials, New York, IEEE Press, 529 pp., 1996. 